\title{
Morning brain: real-world neural evidence that high school class times matter
}

\section{Suzanne Dikker, ${ }^{1,2,}{ }^{*}$ Saskia Haegens, ${ }^{3,4,}{ }^{*}$ Dana Bevilacqua, ${ }^{1,2}$ Ido Davidesco, ${ }^{2}$ Lu Wan, ${ }^{5}$ Lisa Kaggen, ${ }^{2}$ James McClintock, ${ }^{6}$ Kim Chaloner, ${ }^{7}$ Mingzhou Ding, ${ }^{5}$ Tessa West, ${ }^{2}$ and David Poeppel ${ }^{1,2,8}$}

${ }^{1}$ Max Planck-NYU Center for Language, Music and Emotion, New York, NY, USA, ${ }^{2}$ Department of Psychology, New York University, New York, NY, USA, ${ }^{3}$ Department of Psychiatry, Division of Systems Neuroscience, Columbia University and the Research Foundation for Mental Hygiene, New York State Psychiatric Institute, New York, NY, USA, ${ }^{4}$ Donders Institute for Brain, Cognition and Behaviour, Radboud University Nijmegen, The Netherlands, ${ }^{5}$ J. Crayton Pruitt Family Department of Biomedical Engineering, University of Florida, Gainesville, FL, USA, ${ }^{6}$ Trever Day School, New York, NY, USA, ${ }^{7}$ Grace Church School, New York, NY, USA, and ${ }^{8}$ Max Planck Institute for Empirical Aesthetics, Frankfurt am Main, Germany

Correspondence should be addressed to Suzanne Dikker, E-mail: suzanne.dikker@nyu.edu

* These authors contributed equally to this work

\begin{abstract}
Researchers, parents and educators consistently observe a stark mismatch between biologically preferred and socially imposed sleep-wake hours in adolescents, fueling debate about high school start times. We contribute neural evidence to this debate with electroencephalogram data collected from high school students during their regular morning, mid-morning and afternoon classes. Overall, student alpha power was lower when class content was taught via videos than through lectures. Students' resting state alpha brain activity decreased as the day progressed, consistent with adolescents being least attentive early in the morning. During the lessons, students showed consistently worse performance and higher alpha power for early morning classes than for mid-morning classes, while afternoon quiz scores and alpha levels varied. Together, our findings demonstrate that both class activity and class time are reflected in adolescents' brain states in a real-world setting, and corroborate educational research suggesting that mid-morning may be the best time to learn.
\end{abstract}

Key words: real-world neuroscience; classroom learning; hyperscanning; alpha oscillations; portable EEG; circadian rhythms; school times; chronobiology

\section{Introduction}

Among all the ups and downs associated with the high school experience, most readers will distinctly recall trying (and failing) to make it to school on time, especially during the winter season. Most younger kids are early risers, but something drastic happens to sleep/wake patterns around the onset of puberty (Wolfson and Carskadon, 1998; Roenneberg et al., 2004; Crowley et al., 2007): as children enter adolescence, they tend to shift toward later bedtimes. While variations in chronotypes 
exist across all ages, this sharp change in circadian rhythms toward 'eveningness' is so consistent and distinct that it is often regarded as a reliable biomarker for the onset of adolescence (Carskadon et al., 1998, 2006; Dewald et al., 2010; Preckel et al., 2011; Crowley et al., 2014), resulting in a stark mismatch between biologically preferred and socially imposed sleep-wake hours (Crowley et al., 2007; Dewald et al., 2010; Wahlstrom et al., 2014; Kelley et al., 2017) for most teenagers. In fact, chronically inadequate sleep in adolescents is now considered a public health epidemic (Wheaton et al., 2016).

It is well known that low levels of alertness resulting from sleep-related factors are associated with a reduction in the ability to focus, which, in turn, affects learning and task performance (Chee and Choo, 2004; Lim and Dinges, 2010; Sievertsen et al., 2016). To highlight just one example, performance accuracy in adults on certain basic cognitive and neurobehavioral tasks is more impaired after mild sleep deprivation than after alcohol intake that exceeds the levels for legal intoxication (Lamond and Dawson, 1999). For teenagers, various factors connected to sleep (e.g. sleep time, circadian rhythms and sleep loss) are shown to influence levels of focus, alertness and mood, both within and outside of laboratory contexts (Wolfson and Carskadon, 1998; Preckel et al., 2011; Short et al., 2013; Goldin et al., 2020).

Although it can be difficult to disentangle whether low levels of alertness in the morning in high schoolers are due to sleep loss or chronotype (i.e. either adolescents go to bed late and have slept only a few hours by the time they have to wake up, or they are woken up at a bad moment in their natural sleep cycle), the overall observation is that adolescents are less alert in the morning than other age groups and that this lack of alertness leads to a reduction in (cognitive) performance in the morning. Perhaps most strikingly, it has been reported that car accidents in $16-19$-year-olds decreased by $65-70 \%$ when school start time was delayed from 7:55 to 8:25 am (Wahlstrom et al., 2014).

In light of these findings, it is unsurprising that studies consistently find that age-dependent circadian rhythms (as well as chronotype) predict school experience and academic performance (Dewald et al., 2010; Preckel et al., 2011; Short et al., 2013; Vollmer et al., 2013; Wahlstrom et al., 2014). For example, research has shown that sleep-restricted adolescents exhibit poorer cognitive performance and higher levels of sleepiness in the early morning (Short et al., 2013; Lo et al., 2016). As a rather unfortunate coincidence, adolescents spend a sizeable chunk of their mornings attempting to retain information. This has sparked an ongoing public debate about high school start times. In fact, delaying school start times by even 50 min appears to have a significant positive effect on student achievement (Carrell et al., 2011; also see Minges and Redeker, 2016; Bowers and Moyer, 2017). However, effect sizes in these data are often small, and results are largely dependent on explicit selfreport measures (including parental reports; Dewald et al., 2010), which are not always reliable. One possible additional source of information may come from (neuro)physiological data, which has been suggested to constitute a better predictor of behavior than self-report in some cases (Berkman and Falk, 2012; Thorson et al., 2018). With the advance of wearable technology, researchers are now able to collect implicit biophysiological data during everyday activities (Debener et al., 2012), including sleep (de Zambotti et al., 2016). For example, a recent study used wearable activity trackers to assess the relationship between sleep patterns and academic performance in college students (Okano et al., 2019).
Here, we extend on this body of work by focusing on neurophysiological data collected from teenagers as an implicit, real-time measure of their alertness during naturalistic daytime activities. Specifically, we recorded electroencephalogram (EEG) from high school students during their regular classes (consisting of a combination of teacher-led lectures and educational videos) throughout the school day (early morning, mid-morning and afternoon) in two different New York City high schools (Figure 1; Bevilacqua et al., 2019; Dikker et al., 2017).

Although EEG is often employed as a measure of focus/ alertness (Corsi-Cabrera et al., 1992; Cajochen et al., 1995; Horne and Baulk, 2004; Lockley et al., 2006), to our knowledge, we are the first to do so in a real-world school environment. This approach uniquely positioned us to investigate how student brain activity varies as a function of class time and how such neural changes might relate to self-reported focus and academic performance. We focused our analysis on students' brain activity in the alpha frequency range $(\sim 10 \mathrm{~Hz})$. The alpha rhythm, traditionally associated with cortical idling (Adrian and Matthews, 1934; Pfurtscheller et al., 1996), is typically negatively correlated with selective attention and is thought to reflect a mechanism of active inhibition, such that increased alpha activity functions to suppress (distracting) input whereas decreased alpha facilitates processing (Klimesch et al., 2007; Jensen and Mazaheri, 2010; Haegens et al., 2011a 2011b). In addition to reflecting local taskrelated focus, a person's global alpha power is strongly related to overall vigilance or attentiveness (Haslum and Gale, 1973; Linkenkaer-Hansen et al., 2004; Lakatos et al., 2016; Crunelli et al., 2018). We studied how alpha power varied over the course of the day and during different class activities at the group level, comparing alpha activity during teacher-led activities (lectures), educational videos and rest (silently looking at the wall for $2 \mathrm{~min}$ ).

Furthermore, we linked brain activity to both self-reported attentiveness ('how focused are you right now?') and quiz scores. We hypothesized that alpha power would be highest early in the morning and inversely related to students' quiz scores (Preckel et al., 2011). In addition, following previous findings that reported that students are more engaged during videos than lectures (Dikker et al., 2017; Bevilacqua et al., 2019), we predicted that students would exhibit higher alpha power during lectures than videos.

\section{Methods}

We collected EEG and behavioral data from 22 students at two New York City high schools in their senior biology class setting. Here we briefly describe the approach; a more extensive description of the experimental design, recording procedures and preprocessing steps can be found in a previous publication (Dikker et al., 2017).

\section{Participants}

Subjects were 22 healthy high school students (age 17-18 years; 10 students from School 1: 7 females and 3 males, 12 from School 2: 7 females and 5 males) with no known history of neurological disease. All participants provided written informed consent after receiving a detailed explanation of the experimental procedures. For those students who were under 18-years old at the time of data collection, informed consent was also obtained from their legal guardian. The Institutional Review Board of New York University approved all experimental procedures, and 

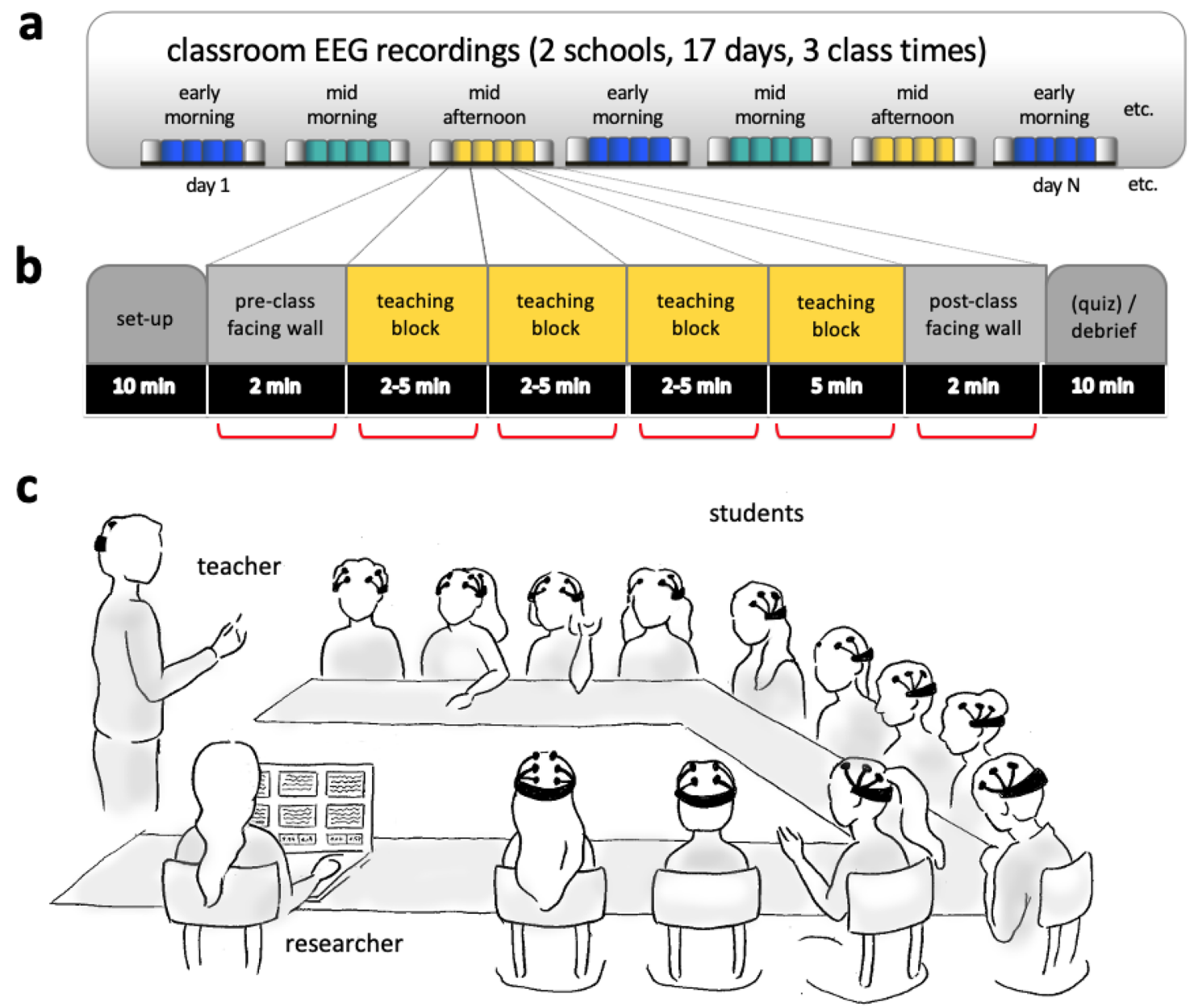

Fig. 1. Experimental setup. (A) Study timeline. EEG activity was recorded from 2 groups of 12 students at 2 separate high schools. Seventeen recording days were scheduled throughout the semester (school 1, 11 recording days; school 2, 6 recording days). Recording sessions were equally distributed across three different class times: early morning, mid-morning and mid-afternoon (AM1, AM2, PM). (B) Experimental procedure of a typical recording day. EEG activity was recorded during four teaching blocks in addition to a resting state segment where students were facing the wall. Lecture and video teaching activities were consistently administered during all 17 recording days across both schools; other tasks varied between days (see, for details, Dikker et al., 2017; Bevilacqua et al., 2019). Student alpha power activity was averaged for each student during each class activity separately (marked in red). (C) Illustration of experimental setup in the classroom with 12 students wearing the EMOTIV EPOC headset. These portable devices offer a rich opportunity to involve students both as participants and as experimenters (Dikker et al., 2017; Bevilacqua et al., 2019).

all methods were carried out in accordance with the relevant guidelines and regulations.

\section{Procedure}

EEG data were recorded during students' regular biology classes over the course of 17 days during the 2014-2015 school year (School 1: 11 classes) and the 2015-2016 school year (School 2: 6 recording days), respectively. Classes were taught at three different class times: early morning (AM1 classes; School 1 start time: 8:30 am, School 2 start time: 9:00 am), mid-morning (AM2 classes; School 1 start time: 10:30 am, School 2 start time: 10:40 am) and mid-afternoon (PM classes; School 1 start time: 2:20 pm, School 2 start time: 2:00 pm). Each class at each school was subdivided into different recording blocks (Figure 1). This included a pre-class rest period where students looked at the wall in silence for $2 \mathrm{~min}$, followed by four teaching blocks (School 1: teacher reads aloud, students watch educational video, teacher lectures and group discussion Dikker et al., 2017; School 2: teacher lectures, students watch educational video, teacher lectures and students watch educational video Bevilacqua et al., 2019), followed by a post-class rest period where students again looked at the wall in silence for $2 \mathrm{~min}$. In both schools, students' self-reported focus level was recorded both before and after class ('How focused do you feel right now?' on a 1-7 scale), and in School 2, students additionally completed a multiple-choice quiz after each class, on content taught during that session. Each quiz consisted of 20 multiple choice questions, with an equal number of questions probing content presented in each of the four teaching blocks (i.e. 5 questions for the first lecture, five questions for the first video, five questions for the second lecture and five questions for the second video). Recordings at different class times never took place on the same day.

\section{Data acquisition}

We used 14-electrode EMOTIV EPOC wireless EEG headsets (mastoid reference locations) to record simultaneous EEG activity from the students in their regular classroom. We used 
custom software based on the OpenFrameworks C++ library (openframe works.com) to simultaneously record the data from all students (Dikker et al., 2017).

\section{Data analysis}

The data were analyzed using custom-built Matlab code, in addition to the EEGLAB (Delorme and Makeig, 2004) and FieldTrip toolboxes (Oostenveld et al., 2011). Continuous recordings were band-pass filtered between 0.5 and $35 \mathrm{~Hz}$ and then segmented into $1 \mathrm{~s}$ epochs. Bad channels and data segments were rejected upon visual inspection. The analysis included on average $15 \mathrm{~min}$ of data during rest (s.d. $=6 \mathrm{~min}), 23 \mathrm{~min}$ for lecture (s.d. $=8 \mathrm{~min}$ ) and $20 \mathrm{~min}$ for video conditions (s.d. $=8 \mathrm{~min}$ ) per subject. For SNR purposes, here we exclusively focused on data recorded with the two occipital electrodes.

\section{Spectral analysis}

Power spectra (4-30 Hz) were computed using a fast Fourier transform approach. The $1 \mathrm{~s}$ epochs were padded with 0-10 s length and multiplied with a Hanning taper, to improve alpha peak frequency detection. For each subject, we computed the average power across all frequencies per recording day (i.e. all conditions combined), per electrode. In order to normalize the data, we divided the single-epoch spectra of the respective day/electrode by this average power value, resulting in relative power values. This procedure reduces variability in the power estimates across electrodes, recording days and subjects. Subsequently, the power spectra were averaged per condition of interest.

\section{Alpha peak detection}

To determine the subject's peak occipital alpha frequency, we detected the highest local maximum within the $7-14 \mathrm{~Hz}$ band with a $0.1 \mathrm{~Hz}$ step size (Haegens et al., 2014), in the average spectrum for that subject (i.e. all occipital electrodes, all recording days, all conditions combined). All subsequent alpha power analysis was performed using a $1 \mathrm{~Hz}$ band centered at this individual peak frequency. As a control analysis, we verified that there were no significant differences in alpha peak frequency across conditions (repeated measures ANOVA with factor class condition: $F(2,42)=0.657, P=0.523)$ or time of day $(F=0.765$, $P=0.472)$.

\section{Statistical analysis}

To analyze students' alpha power and focus over the course of the day, data from both schools were combined $(N=22)$. To adjust for non-independence over repeated measures, participants' pre- and post-session resting state alpha power was compared to self-reported focus scores using multilevel modeling, using the MIXED procedure in SPSS, specifying an unstructured correlation structure (in which variances for each repeated measure and all possible covariances are estimated). The 'time' repeated measure factor was analyzed in two ways. First, the 'pre/post' time factor evaluated resting state relative alpha power and focus ratings before and after lessons, regardless of time of day. Second, the time factor was analyzed as 'time of day' for alpha power, focus ratings and quiz scores per teaching style for the sessions' time of day occurrence (i.e. AM1 us AM2 us PM).

For the second school $(N=12)$, we analyzed students' alpha power in relation to performance via post-lesson quizzes for both teaching methods (i.e. lectures and videos; this information was not collected for the first school). In this analysis, we adjusted for non-independence of repeated observations nested within students using GEE (Ballinger, 2004). The distribution of the response variable was specified as normal, and an unstructured correlation matrix was specified (in which all possible correlations between within-subjects' responses are estimated (Fitzmaurice et al., 1993). Commonly, this specialized model applies to complex data structures that account for missing data, multiple regression parameters and repeated measure within-subject correlations (Westgate and Burchett, 2017). This model was computed via the GENLIN command in SPSS.

\section{Results}

We recorded students' EEG before class ('resting state' or no task), during class (lectures and videos) and after class (postclass resting state; see Figure 1). In addition, we collected behavioral measures from the students, including self-reported focus scores and quiz performance for each class. We determined each student's individual alpha peak frequency (based on overall power spectra for occipital electrodes; $M=9.56 \mathrm{~Hz}$, s.d. $=0.85$; Figure 2A), and used this for all power analyses described below.

\section{Alpha power varies by class activity}

As an initial step, we asked whether students' alpha power varied as a function of class activity (pre- and post-class resting states, lectures and videos). We found that alpha power was significantly decreased during class compared to resting state, with lower alpha power during videos compared to lectures (repeated measures analysis of variance (ANOVA) with factor class condition: $F(2,42)=27.94, P<0.001$, post-hoc correct pairwise comparisons all $\mathrm{P}<0.01$; Figure $2 \mathrm{~B}-\mathrm{C}$ ).

\section{Theta power does not vary by class activity}

For the purpose of this study, we were specifically interested in posterior alpha activity. However, frontal theta activity $(4-7 \mathrm{~Hz})$ would make another obvious focus of interest, since it has previously been associated with focus/attention (Cajochen et al., 1995). While the power spectra showed no discernible theta modulation, neither on the single subject level nor averaged across subjects (in contrast to the very clear alpha peaks; Figure 2), as a sanity check, we nevertheless repeated our main effect analysis for frontal theta activity. We found no significant modulation of theta power by class activity $(F(2,42)=0.693$, $P=0.51$ ), suggesting specificity of our results to the alpha band.

\section{Alpha power during rest decreases throughout the day}

To evaluate whether students' occipital alpha power varied as a function of class time, we first looked at students' EEG activity in the absence of a learning task (i.e. pre- and post-class resting states). All statistics reported below were derived using multilevel modeling in SPSS using the MIXED procedure to adjust for non-independence in repeated measures of alpha measures within student over time (see Hox et al., 2018; we note that the MIXED procedure can yield fractional degrees of freedom because they are computed using the Satterthwaite method (Fitzmaurice et al., 2004)). Given that each student provided data for three time points, we included the random effect of 

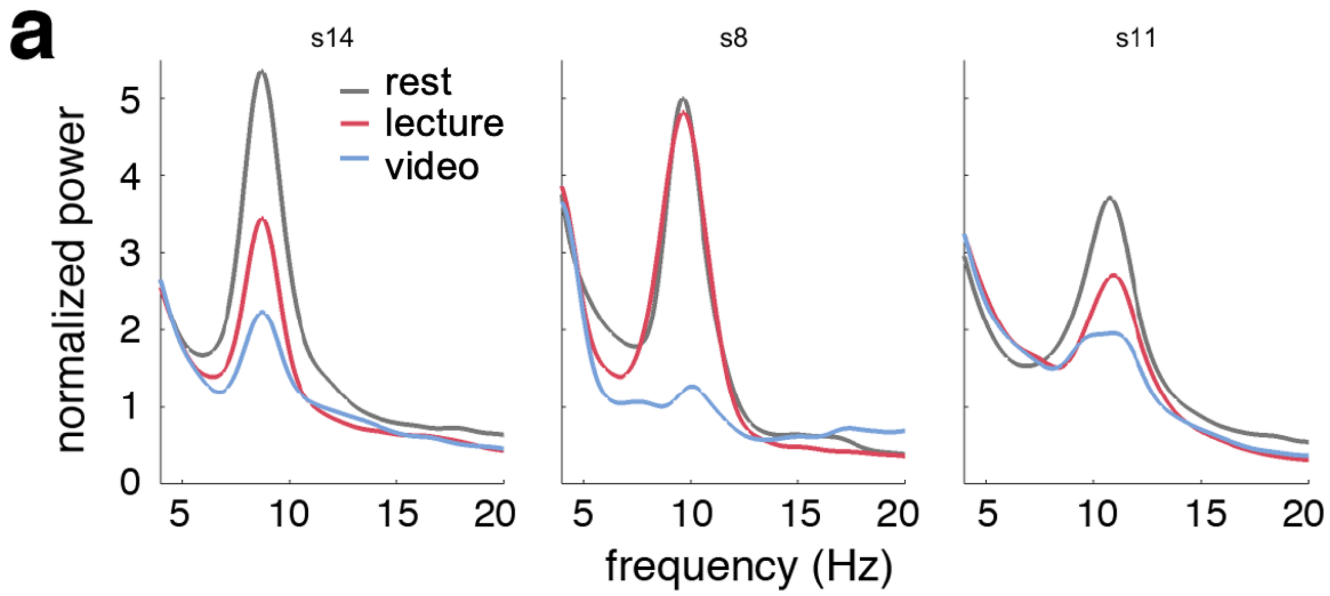

b
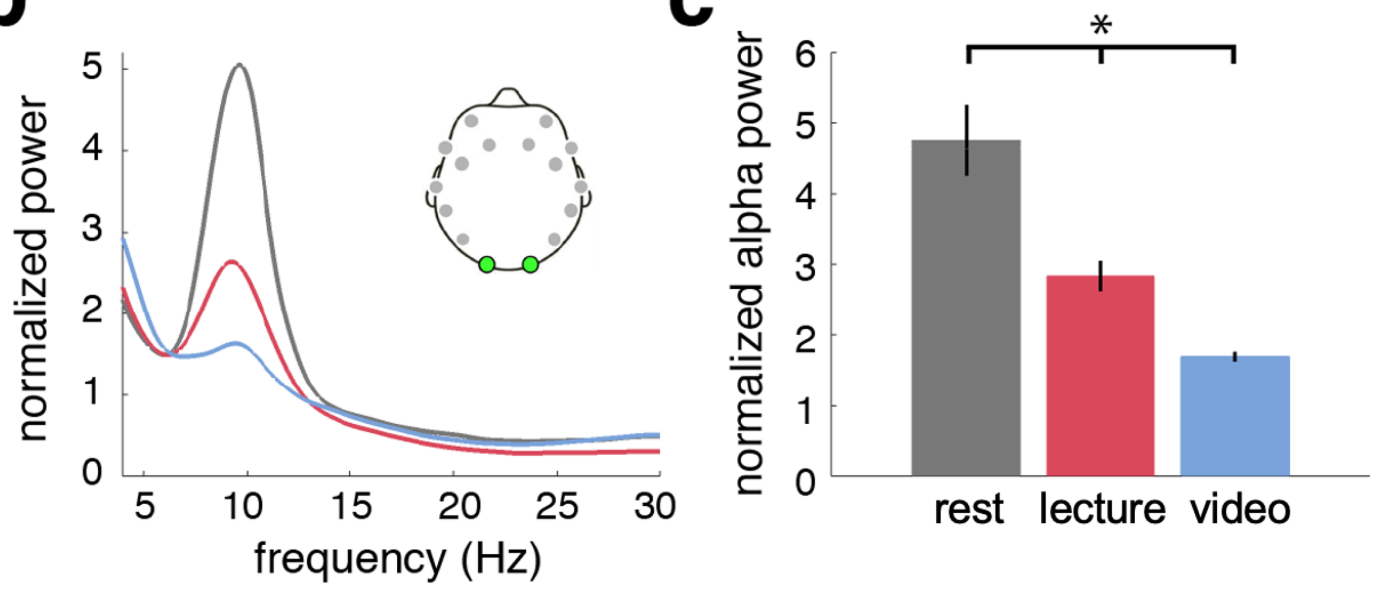

Fig. 2. Alpha power per condition. (A) Power spectra (averaged over occipital sensors, see inset in panel B) of three representative example subjects for each of the conditions of interest (resting state in gray, lecture in pink and video in blue), showing clear peaks in the alpha band. (B) Similar as in A, averaged over all subjects $(\mathrm{N}=22)$, including all available recording sessions per subject. (C) A significant $(P<0.001)$ decrease of posterior alpha power over conditions (computed in 1 Hz band centered at individual alpha peak frequency for each subject, normalized with average power in the spectrum per channel). Error bars indicate standard error of the mean (SEM; $N=22)$.

each time point early morning, mid-morning and afternoon and all covariances between time points, resulting in a saturated random effects model (AM1 with AM2, AM1 with PM and AM2 with PM); all random effects, Ps $<0.001)$. We found that students' alpha power significantly differed across class times for resting state recordings with the highest alpha power during early morning classes (all MIXED procedure SPSS; AM1 Us AM2 us PM: $F(2,21.16)=4.34, P=0.026)$. Alpha power declined as the day progressed, with the highest alpha power for pre-class resting state during early morning classes (AM1>PM; Figure 3 ). In addition, alpha power was significantly lower during post-class resting state recordings than pre-class resting state recordings, independent of class time (pre us post: $F(1,16.88)=19.99$, $P<0.001)$. Students' self-reported focus did not vary significantly as a function of class time (AM1 us AM2 us PM: $F(2,20.55)=0.50$, $P=0.61$.

\section{Lowest alpha power and highest quiz scores for mid-morning classes}

Next, we turned to student learning as a function of class time. Since quiz scores were not available for School 1, all data reported below pertain to School 2. Data were analyzed using Generalized Estimating Equations (GEE), an approach suited for testing repeated measures within subjects across time (Ballinger, 2004). GEE was developed by Zeger and Liang (1986) as a means of testing hypotheses regarding data that are collected within subjects across repeated measures. GEE model the mean response and the within-subject response separately; thus, the interpretation of the regression parameters is not altered by assumptions made about the nature and magnitude of the within-subject association.

In our GEE analysis, we specified a continuous outcome variable (quiz score), and an unstructured correlation matrix, in which all possible correlations between within-subjects' responses are adjusted for (ideal here because quiz scores are likely correlated within subject, across different quizzes; Fitzmaurice et al., 1993). A main effect of time of day (AM1, AM2, PM) was found (Wald $\chi^{2}(2)=23.377, P<0.001$; Figure $4 A$ ). As seen in Figure $4 \mathrm{~A}$, the average quiz score at AM2 was significantly higher than the average score at AM1 (Wald $\chi^{2}(1)=17.79$, $P<0.001$ ) and afternoon classes(Wald $\chi^{2}(1)=10.07, P=0.002$ ). The average score at AM1 did not differ from the average score at PM (Wald $\chi^{2}(1)=0.088, P=0.766$ ). 


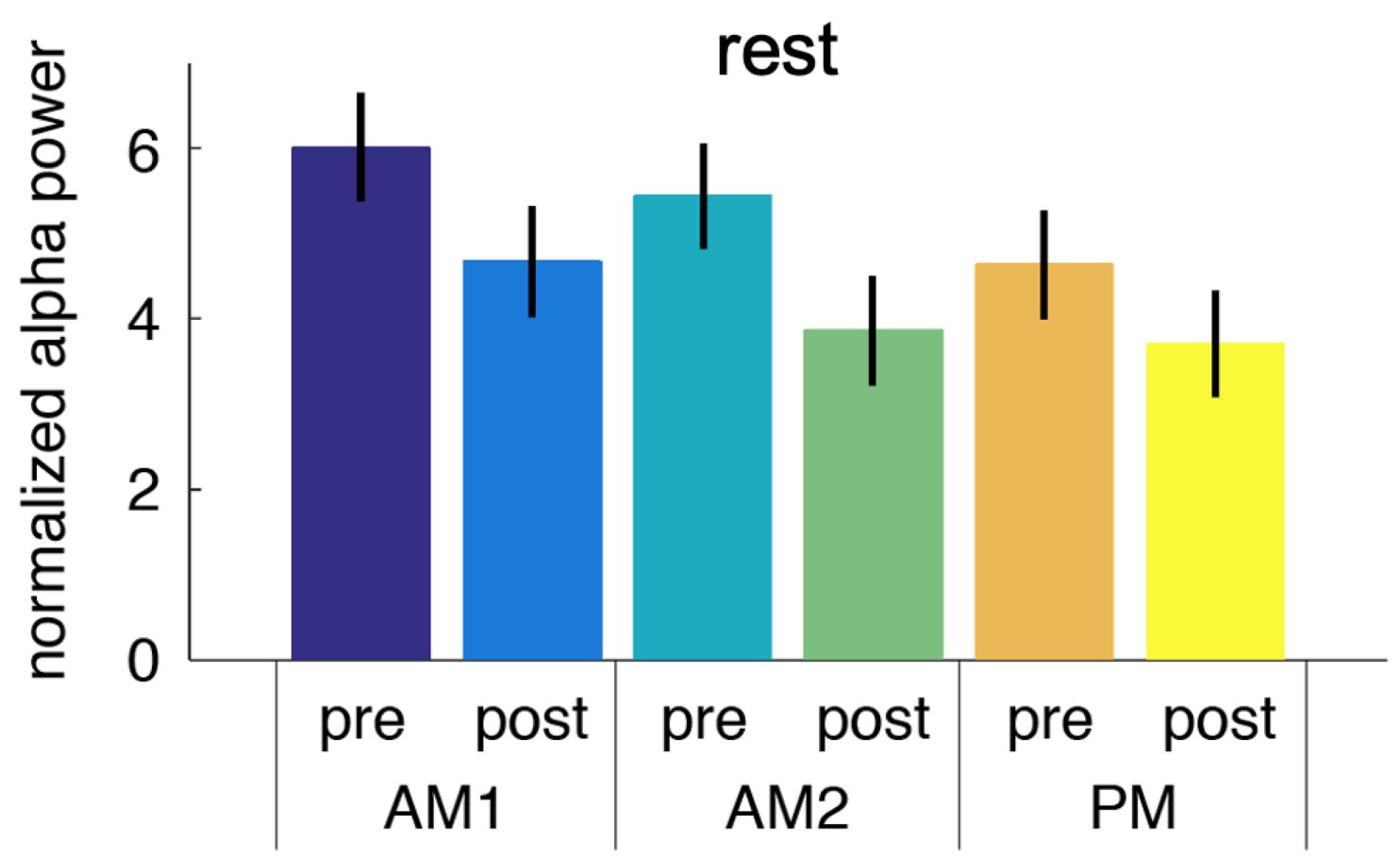

Fig. 3. Alpha power and focus over the course of the day. Resting state posterior alpha power, measured bot 10 before and after class (computed in $1 \mathrm{~Hz}$ band centered at individual alpha peak frequency for each subject, normalized with average power in the spectrum per channel) significantly decreased with later class times $(P<0.001)$ : we observed the highest alpha power at the beginning of the early morning classes and the lowest alpha power at the end of the afternoon classes. Showing data for all subjects combined $(\mathrm{N}=22)$. [AM1: early morning session, AM2: mid-morning session, PM: afternoon session. Error bars indicate SEM.].
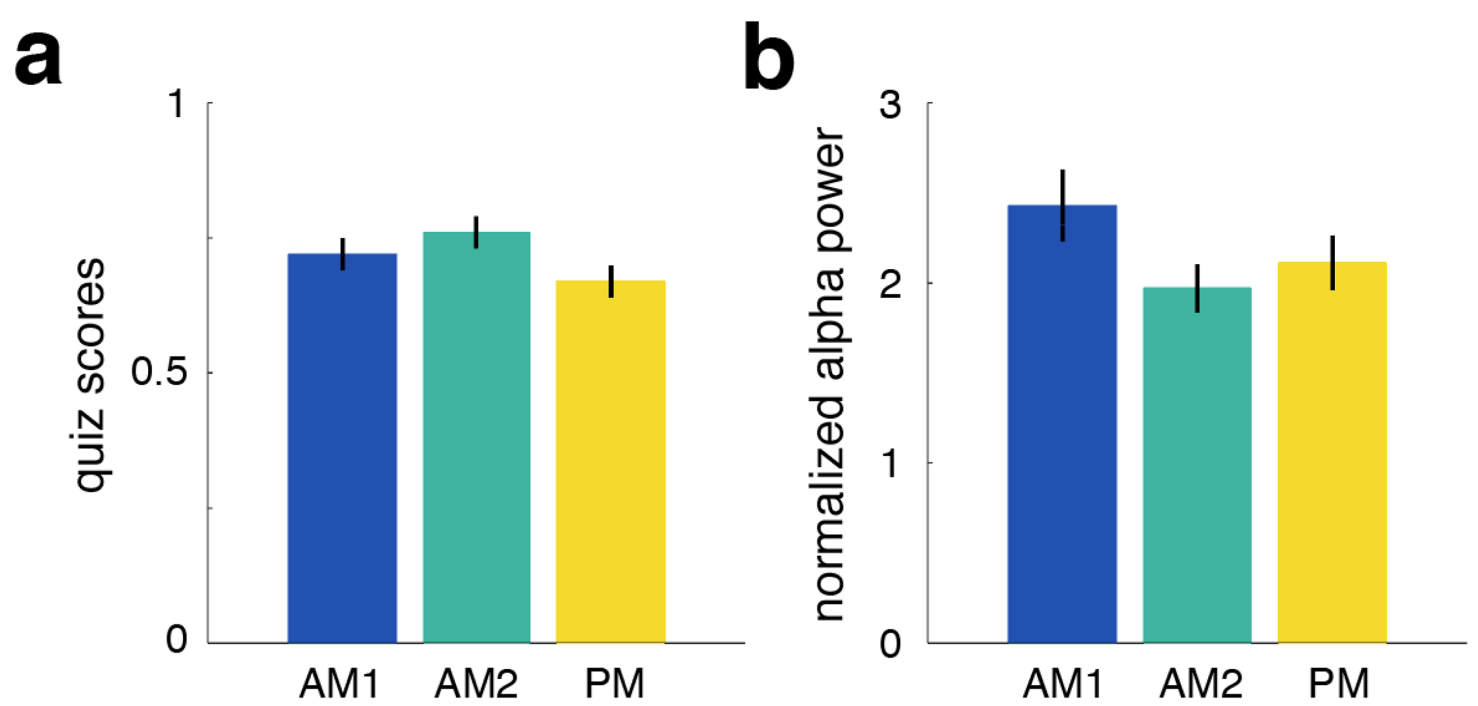

Fig. 4. Alpha power and performance. (A) Quiz scores were highest for mid-morning classes. (B) Alpha power was highest for early-morning classes $(P=0.05)$. [AM1: early morning session, AM2: mid-morning session, PM: afternoon session. Error bars indicate SEM.].

For alpha power, a main effect of time of day was also found (Wald $\chi^{2}(2)=6.073, P=0.048$; Figure $4 B$ ). As seen in Figure 4B, students exhibited significantly lower alpha power in the early morning classes relative to mid-morning (Wald $\chi^{2}(1)=5.985$, $P<0.001$ ) and afternoon classes (Wald $\chi^{2}(1)=10.07, P=0.002$ ). Similar to the quiz score results, alpha power in the early morning was not significantly different from alpha power in the afternoon (Wald $\chi^{2}(1)=0.971, P=0.324$ ). In addition, alpha power in the early morning did not significantly differ from alpha power in the afternoon (Wald $\chi^{2}(1)=1.490, P=0.222$ ).
In sum, quiz scores and student alpha power both varied by class time. Alpha power was consistently highest during early morning classes. Quiz scores were highest, and alpha activity was lowest, during mid-morning classes. Resting state alpha power (when students were facing the wall without a task) continued to drop in the afternoon. During class activities, in contrast, alpha rose up again in the afternoon compared to midmorning. Exploratory analyses assessing alpha power effects by class activity and by individual differences are discussed in the Supplemental Materials. 


\section{Discussion}

We collected EEG data from two classes of high school seniors at two different New York City schools during their regular biology lessons. Classes were taught at three different times of day: early morning, mid-morning and afternoon (17 recording days total). To investigate student attentiveness at school, we examined group-level changes in power in the alpha frequency band $(\sim 7-14 \mathrm{~Hz})$, a well-known correlate of attentional state (Klimesch et al., 2007; Jensen and Mazaheri, 2010; Haegens et al., 2011b), where higher alpha power is typically linked to lower focus and decreased performance (Thut et al., 2006; Haegens et al., 2011a).

\section{Alpha varies with lesson activity}

First and foremost, we observed a robust difference in alpha power during different class activities: student's alpha activity was lowest while they were watching educational videos, significantly higher during lectures, and highest during rest. This matches the well-known effect of alpha power generally decreasing with engagement (Adrian and Matthews, 1934; Pfurtscheller et al., 1996). Quiz scores as well as student engagement ratings were also higher for videos than lectures (reported in Dikker et al., 2017; Bevilacqua et al., 2019). Together, these findings are consistent with an account whereby highly engaging stimuli increase shared attention among viewers, which in turn reduces alpha power at the group level. (See Dikker et al. (2017) for data and a discussion pertaining to how a decrease in alpha power as a function of classroom engagement may be coupled with an increase in EEG similarity, or brain-to-brain synchrony, between classmates.) These effects are likely driven by a combination of low-level stimulus features (Cohen and Parra, 2016; Ki et al., 2016; Poulsen et al., 2017) as well as high-level (social/discourse-related) factors (Stephens et al., 2010; Koike et al., 2016): processing attended stimuli reduces alpha activity (Ward, 2003; Klimesch et al., 2007) and increases entrainment (Zion Golumbic et al., 2013).

Before concluding that students should just be passively watching videos all day however (Freeman et al., 2014; Hew Khe, 2014) it is important to note that the lectures were not necessarily a fair representation of the richness of the typical face-to-face teaching experience. For example, teachers in both schools were instructed to keep movement to a minimum, to remain seated and to refrain from using visual support for their lectures. Furthermore, students were asked to keep questions about the material to themselves until the lecture portion was finished. All in all, then, the lectures were perhaps more dull than what the students would have experienced in their day-to- day classes.

At first glance, the reduction in alpha power after class (lower post-class resting state alpha than pre-class resting state alpha) seems incompatible with an attention explanation (in typical laboratory experiments, alpha increases over the course of a task; Benwell et al., 2017). Although we can only speculate why this was the case, one possible explanation is task-related. It is not unlikely that during the post-class rest period, students were rehearsing content they had just been presented during classes, in preparation for the post-class survey where they would be asked to recall and/or reflect on the class content and their class experience. Some indirect support for this explanation comes from findings suggesting that spontaneous thinking during resting states engages similar neural networks activated in problem-solving states (Kounios et al., 2008).

\section{Alpha varies with class time}

Now let's turn to the main focus of this study-student alpha power in relation to class time. First, students' alpha power during rest periods decreased as the time of day progressed, in line with prior work suggesting that students become more alert and less sleepy throughout the day (at least until the midafternoon). When students were presented with class content, however, a slightly different pattern emerged: student alpha power was lowest during the mid-morning classes, which mirrored their performance: retention was highest for materials learned in mid-morning classes. This finding is consistent with previous research on student performance that also shows such a 'U-shaped' relationship to time of day. This seemingly internally contradictory finding may well result from a combination of circadian factors and fatigue: early morning performance is found to be low because of circadian reasons (Preckel et al., 2011), whereas performance on tests taken at the end of a school day is argued to be low because of fatigue/saturation (Dewald et al., 2010; Short et al., 2013).

This relates to the fact that both global alpha power and selfreported focus both lack specificity: they likely reflect multiple mental states, closely related yet distinct. First, as noted in the Introduction and discussed above, alpha power is associated not only with local (or selective) task-related attention allocation (stimulus engagement) but also with global attentiveness (brain states) -in both cases lower alpha corresponds to higher levels of attention. (Note that increased local alpha is thought to be associated with active selective suppression of distracting inputs-i.e. the counterpart of attention; however, with the alpha measures, we are reporting here, we are likely not picking up on such more nuanced effects). Thus, our findings may very well reflect a combination of both local and global processes.

Most prior research investigating effects of circadian rhythms on school performance, motivation and engagement has targeted individual variation among adolescents in terms of e.g. their chronotype and/or hours of sleep (Curcio et al., 2006; Dewald et al., 2010; Preckel et al., 2011; Roeser et al., 2013; Short et al., 2013; Vollmer et al., 2013; Okano et al., 2019; Goldin et al., 2020). Unfortunately, we did not have access to this information from the students. In lieu, we had to rely on a fairly crude measure of self-reported focus, for which students received no additional instructions beyond the question 'how focused do you feel right now?'. While our supplementary analyses tentatively support prior findings that individual variation may differentially affect alertness, mood and academic performance (Roeser et al., 2013; Short et al., 2013; Vollmer et al., 2013), we observed no main effect of class time on self-reported focus. One of various possible explanations is that in the early morning, students equated lack of focus with sleepiness (i.e. global states of attentiveness), while in the afternoon they associated it more often with distractibility (i.e. local stimulus engagement): maybe they just broke up with a boyfriend, they may have heard back from their college applications, perhaps they just finished a difficult chemistry test, etc. Whichever the underlying cause, this null result underscores the relevance of implicit measures such as brain imaging in conjunction with self-report and behavioral metrics (see e.g. Thorson et al., 2018 for a more general argument about the problematic nature of self-report).

Finally, we did not replicate previous findings showing that frontal theta activity is associated with sustained wakefulness (Cajochen et al., 1995). Note, however, that the lack of frontal theta activity in our data could simply be a result of low signalto-noise ratio, possibly because the recording setup might be 
more sensitive to the prominent posterior alpha rhythm; hence, this does not allow us to draw any conclusions about the potential relevance or role of frontal theta in classroom engagement.

\section{Conclusion}

Together, our findings demonstrate that class time is reflected in adolescents' brain state and suggest that mid-morning may be the best time to learn. Perhaps most importantly, we validated that non-invasive neurophysiological measures can be employed as an implicit, continuous measure of attentiveness during real-world classroom learning.

\section{Acknowledgements}

We thank the school staff and especially the AP Biology students for all of their time, support and enthusiasm; Hasibe Melda Kahraman and Jess Rowland for their research assistance, and Tina Sundelin as well as four anonymous reviewers for their very helpful comments on a previous version of the manuscript.

\section{Conflicts of Interest}

The authors declare that they have no competing interests.

\section{Funding}

This research was supported by Netherlands Organization for Scientific Research VENI Awards 275-89-018 (SD) and 451-14-027 (SH), NSF INSPIRE Track 1 Award 1344285 (DP), and NSF ECR Award 1661016 (DP).

\section{Author contributions}

$\mathrm{SD}, \mathrm{DB}, \mathrm{LK}, \mathrm{LW}, \mathrm{JM}, \mathrm{KC}, \mathrm{MD}$ and $\mathrm{DP}$ designed the research; SD, DB, LK, ID, JM, and KC conducted research; SH, DB, LW, SD, and TW performed data analysis; SD, SH, DB, TW, and DP wrote the paper.

\section{Supplementary data}

Supplementary data are available at SCAN online.

\section{References}

Adrian, E.D., Matthews, B.H.C. (1934). The Berger rhythm: potential changes from the occipital lobes in man. Brain, 57(4), 355-85.

Ballinger, G.A. (2004). Using generalized estimating equations for longitudinal data analysis. Organizational Research Methods, 7(2), 127-50.

Benwell, C.S.Y., Keitel, C., Harvey, M., Gross, J., Thut, G. (2017). Trial-by-trial co-variation of pre-stimulus EEG alpha power and visuospatial bias reflects a mixture of stochastic and deterministic effects. European Journal of Neuroscience, 111, 1300-19.

Berkman, E.T., Falk, E.B. (2012). Beyond brain mapping: using neural measures to predict real-world outcomes. Current Directions in Psychological Science, 22(1), 45-50.
Bevilacqua, D., Davidesco, I., Wan, L., et al. (2019). Brainto-brain synchrony and learning outcomes vary by studentteacher dynamics: evidence from a real-world classroom electroencephalography study. Journal of Cognitive Neuroscience, 31(3), 401-11.

Bowers, J.M., Moyer, A. (2017). Effects of school start time on students' sleep duration, daytime sleepiness, and attendance: a meta-analysis. Sleep Health, 3(6), 423-31.

Cajochen, C., Brunner Dp Fau - Krauchi, K., Krauchi K Fau - Graw, P., Graw P Fau - Wirz-Justice, A., Wirz-Justice, A. (1995). Power density in theta/alpha frequencies of the waking EEG progressively increases during sustained wakefulness. Sleep, 18(10), 890-94.

Carrell, S.E., Maghakian, T., West, J.E. (2011). A's from Zzzz's? The causal effect of school start time on the academic achievement of adolescents. American Economic Journal: Economic Policy, 3(3), 62-81.

Carskadon, M.,.A., Acebo, C., Jenni Oskar, G. (2006). Regulation of adolescent sleep: implications for behavior. Annals of the New York Academy of Sciences, 1021(1), 276-91.

Carskadon, M.A., Wolfson, A.R., Acebo, C., Tzischinsky, O., Seifer, R. (1998). Adolescent sleep patterns, circadian timing, and sleepiness at a transition to early school days. Sleep, 21(8), 871-81.

Chee, M.W.L., Choo, W.C. (2004). Functional imaging of working memory after $24 \mathrm{hr}$ of total sleep deprivation. The Journal of Neuroscience, 24(19), 4560.

Cohen, S.S., Parra, L.C. (2016). Memorable audiovisual narratives synchronize sensory and supramodal neural responses. eNeuro, 3(6).

Corsi-Cabrera, M., Ramos, J., Arce, C., Guevara, M.A., Poncede León, M., Lorenzo, I. (1992). Changes in the waking EEG as a consequence of sleep and sleep deprivation. Sleep, 15(6), 550-55.

Crowley, S.J., Acebo, C., Carskadon, M.A. (2007). Sleep, circadian rhythms, and delayed phase in adolescence. Sleep Medicine, 8(6), 602-12.

Crowley, S.J., Van Reen, E., LeBourgeois, M.K., et al. (2014). A longitudinal assessment of sleep timing, circadian phase, and phase angle of entrainment across human adolescence. Plos One, 9(11), e112199.

Crunelli, V., Iôrincz, M.L., Connelly, W.M., et al. (2018). Dual function of thalamic low-vigilance state oscillations: rhythmregulation and plasticity. Nature Reviews. Neuroscience, 19(2), 107-18.

Curcio, G., Ferrara, M., De Gennaro, L. (2006). Sleep loss, learning capacity and academic performance. Sleep Medicine Reviews, 10(5), 323-37.

de Zambotti, M., Godino, J.G., Baker, F.C., Cheung, J., Patrick, K., Colrain, I.M. (2016). The boom in wearable technology: cause for alarm or just what is needed to better understand sleep? Sleep, 39(9), 1761-62.

Debener, S., Minow, F., Emkes, R., Gandras, K., De Vos, M. (2012). How about taking a low-cost, small, and wireless EEG for a walk? Psychophysiology, 49(11), 1617-21.

Delorme, A., Makeig, S. (2004). EEGLAB: an open source toolbox for analysis of single-trial EEG dynamics including independent component analysis. Journal of Neuroscience Methods, 134(1), 9-21.

Dewald, J.F., Meijer, A.M., Oort, F.J., Kerkhof, G.A., Bögels, S.M. (2010). The influence of sleep quality, sleep duration and sleepiness on school performance in children and adolescents: a meta-analytic review. Sleep Medicine Reviews, 14(3), 179-89. 
Dikker, S., Wan, L., Davidesco, I., et al. (2017). Brain-to-brain synchrony tracks real-world dynamic group interactions in the classroom. Current Biology, 27(9), 1375-80.

Fitzmaurice, G.M., Laird, N.M., Rotnitzky, A.G. (1993). Regression models for discrete longitudinal responses. Statistical Science, 8(3), 284-99.

Fitzmaurice, G.M., Laird, N.M., Ware, J.H. (2004). Applied Longitudinal Analysis Hoboken, NJ: John Wiley

Freeman, S., Eddy, S.L., McDonough, M., et al. (2014). Active learning increases student performance in science, engineering, and mathematics. Proceedings of the National Academy of Sciences, 111(23), 8410.

Goldin, A.P., Sigman, M., Braier, G., Golombek, D.A., Leone, M.J. (2020). Interplay of chronotype and school timing predicts school performance. Nature Human Behaviour, 4(4), 387-96.

Haegens, S., Cousijn, H., Wallis, G., Harrison, P.J., Nobre, A.C. (2014). Inter- and intra-individual variability in alpha peak frequency. NeuroImage, 92(0), 46-55.

Haegens, S., Händel, B.F., Jensen, O. (2011a). Top-down controlled alpha band activity in somatosensory areas determines behavioral performance in a discrimination task. Journal of Neuroscience, 31(14), 5197-204.

Haegens, S., Nácher, V., Luna, R., Romo, R., Jensen, O. (2011b). $\alpha$-Oscillations in the monkey sensorimotor network influence discrimination performance by rhythmical inhibition of neuronal spiking. Proceedings of the National Academy of Sciences, 108(48), 19377-82.

Haslum, M.N., Gale, A. (1973). Inter-modal and intra-subject consistency in EEG correlates of vigilance. Biological Psychology, 1(2), 139-50.

Hew Khe, F. (2014). Promoting engagement in online courses: what strategies can we learn from three highly rated MOOCS. British Journal of Educational Technology, 47(2), 320-41.

Horne, J.A., Baulk, S.D. (2004). Awareness of sleepiness when driving. Psychophysiology, 41(1), 161-65.

Hox, J.J., Moerbeek, M., Van de Schoot, R. (2018). Multilevel Analysis: Techniques and Applications, Third Edition. Routledge.

Jensen, O., Mazaheri, A. (2010). Shaping functional architecture by oscillatory alpha activity: gating by inhibition. Frontiers in Human Neuroscience, 4, 186.

Kelley, P., W. Lockley, S., Kelley, J., D. R. Evans, M. (2017). Is 8:30 a.m. still too early to start school? A 10:00 a.m. school start time improves health and performance of students aged 13-16. Frontiers in Human Neuroscience, 11, 588.

Ki, J.J., Kelly, S.P., Parra, L.C. (2016). Attention strongly modulates reliability of neural responses to naturalistic narrative stimuli. The Journal of Neuroscience, 36(10), 3092.

Klimesch, W., Sauseng, P., Hanslmayr, S. (2007). EEG alpha oscillations: the inhibition-timing hypothesis. Brain Research Reviews, 53(1), 63-88.

Koike, T., Tanabe, H.C., Okazaki, S., et al. (2016). Neural substrates of shared attention as social memory: a hyperscanning functional magnetic resonance imaging study. NeuroImage, 125, 401-12.

Kounios, J., Fleck, J.I., Green, D.L., et al. (2008). The origins of insight in resting-state brain activity. Neuropsychologia, 46(1), 281-91.

Lakatos, P., Barczak, A., Neymotin, S.A., et al. (2016). Global dynamics of selective attention and its lapses in primary auditory cortex. Nature Neuroscience, 19(12), 1707-17.

Lamond, N., Dawson, D. (1999). Quantifying the performance impairment associated with fatigue. Journal of Sleep Research, $8(4), 255-62$.
Lim, J., Dinges, D.F. (2010). A meta-analysis of the impact of short-term sleep deprivation on cognitive variables. Psychological Bulletin, 136(3), 375-89.

Linkenkaer-Hansen, K., Nikulin, V.V., Palva, S., Ilmoniemi, R.J., Palva, J.M. (2004). Prestimulus oscillations enhance psychophysical performance in humans. Journal of Neuroscience, 24(45), 10186-90.

Lo, J.C., Ong, J.L., Leong, R.L.F., Gooley, J.J., Chee, M.W.L. (2016). Cognitive performance, sleepiness, and mood in partially sleep deprived adolescents: the need for sleep study. Sleep, 39(3), 687-98.

Lockley, S.W., Evans, E.E., Scheer, F.A.J.L., Brainard, G.C., Czeisler, C.A., Aeschbach, D. (2006). Short-wavelength sensitivity for the direct effects of light on alertness, vigilance, and the waking electroencephalogram in humans. Sleep, 29(2), 161-68.

Minges, K.E., Redeker, N.S. (2016). Delayed school start times and adolescent sleep: a systematic review of the experimental evidence. Sleep Medicine Reviews, 28, 86-95.

Okano, K., Kaczmarzyk, J.R., Dave, N., Gabrieli, J.D., Grossman, J.C. (2019). Sleep quality, duration, and consistency are associated with better academic performance in college students. NPJ Science of Learning, 4(1), 1-5.

Oostenveld, R., Fries, P., Maris, E., Schoffelen, J.-M. (2011). FieldTrip: open source software for advanced analysis of MEG, EEG, and invasive electrophysiological data. Computational Intelligence and Neuroscience, 2011, 156869.

Pfurtscheller, G., Stancak, A., Neuper, C. (1996). Event-related synchronization (ERS) in the alpha band-an electrophysiological correlate of cortical idling: a review. International Journal of Psychophysiology, 24(1-2), 39-46.

Poulsen, A.T., Kamronn, S., Dmochowski, J., Parra, L.C., Hansen, L.K. (2017). EEG in the classroom: synchronised neural recordings during video presentation. Scientific Reports, 7, 43916.

Preckel, F., Lipnevich, A., Schneider, Roberts, R. (2011). Chronotype, cognitive abilities, and academic achievement: a metaanalytic investigation. Learning and Individual Differences, 21(5), 483-92.

Roenneberg, T., Kuehnle, T., Pramstaller, P.P., et al. (2004). A marker for the end of adolescence. Current Biology, 14(24), R1038-R1039.

Roeser, K., Schlarb, A.A., Kübler, A. (2013). The ChronotypeAcademic Performance Model (CAM): daytime sleepiness and learning motivation link chronotype and school performance in adolescents. Personality and Individual Differences, 54(7), 836-40.

Short, M.A., Gradisar, M., Lack, L.C., Wright, H.R. (2013). The impact of sleep on adolescent depressed mood, alertness and academic performance. Journal of Adolescence, 36(6), 1025-33.

Sievertsen, H.H., Gino, F., Piovesan, M. (2016). Cognitive fatigue influences students' performance on standardized tests. Proceedings of the National Academy of Sciences, 113(10), 2621.

Stephens, G.J., Silbert, L.J., Hasson, U. (2010). Speaker-listener neural coupling underlies successful communication. Proceedings of the National Academy of Sciences, 107(32), 14425.

Thorson, K., V. West, T., Mendes, W. (2018). Measuring physiological influence in dyads: a guide to designing, implementing, and analyzing dyadic physiological studies. Psychological Methods, 23(4), 595

Thut, G., Nietzel, A., Brandt, S.A., Pascual-Leone, A. (2006). Alpha-band electroencephalographic activity over occipital cortex indexes visuospatial attention bias and predicts visual target detection. Journal of Neuroscience, 26(37), 9494-502. 
Vollmer, C., Pötsch, F., Randler, C. (2013). Morningness is associated with better gradings and higher attention in class. Learning and Individual Differences, 27, 167-73.

Wahlstrom, K., Dretzke, B., Gordon, M., Peterson, K., Edwards, K., Gdula, J. (2014). Examining the Impact of Later High School Start Times on the Health and Academic Performance of High School Students: A Multi-Site Study. Center for Applied Research and Educational Improvement. St. Paul, MN: University of Michigan.

Ward, L.M. (2003). Synchronous neural oscillations and cognitive processes. Trends in Cognitive Sciences, 7(12), 553-59.

Westgate, P.M., Burchett, W.W. (2017). A comparison of correlation structure selection penalties for generalized estimating equations. The American Statistician, 71(4), 344-53.
Wheaton, A.O., Emily, Miller, G., Croft, J. (2016). Sleep duration and injury-related risk behaviors among high school students-United States, 2007-2013. C. F. D. Control. Ed. Vol. 65. MMWR Morb Mortal Wkly Rep. pp. 337-341.

Wolfson, A.R., Carskadon, M.A. (1998). Sleep schedules and daytime functioning in adolescents. Child Development, 69(4), 875-87.

Zeger, S.L., Liang, K.Y. (1986). Longitudinal data analysis for discrete and continuous outcomes. Biometrics, 42, 121-130.

Zion Golumbic, E.M., Ding, N., Bickel, S., et al. (2013). Mechanisms underlying selective neuronal tracking of attended speech at a "cocktail party". Neuron, 77(5), 980-91. 